\title{
Verhaltenstherapie
}

\section{Band 16, Heft 2, Juni 2006}

\section{Editorial}

85 Neuropsychotherapie: Eine Frage der

Verantwortung?

Hellhammer, D. (Trier)

\section{Übersichtsarbeiten}

86 Neuropsychotherapie bei chronischen Schmerzen: Veränderung des Schmerzgedächtnisses durch Verhaltenstherapie Flor, H.; Hermann, C. (Mannheim)

96 Wiedererfahrung durch Psychotherapie modifziert Geist und Gehirn

Schauer, M.; Elbert, T.; Gotthardt, S.; Rockstroh, B.; Odenwald, M.; Neuner, F. (Konstanz)

104 Neuronale Korrelate der Expositionstherapie bei Patienten mit spezifischen Phobien Schienle, A. (Trier/Gießen); Schäfer, A. (Gießen)

112 Neuropsychologische Diagnostik und Therapie von Aufmerksamkeits- und Gedächtnisstörungen im Kindesalter

Petermann, F.; Lepach, A. (Bremen)

122 Verhaltensmedizin, Psychotherapie und Zahnheilkunde Meinlschmidt, G.; Bolt, O. (Trier/Basel)

Diskussionsforum

133 Neuropsychotherapie: Anmerkungen eines Neuropsychologen Gauggel, S. (Aachen)

Interview

139 Niels Birbaumer: «Der biopsychologische Wissenszuwachs wird die Frage nach der Indikation und Prognose von Psychotherapie revolutionieren» Hautzinger, M. (Tübingen)
Vol. 16, Issue 2, June 2006

\section{Editorial}

85 Neuropsychotherapy: A Question of

Responsibility?

Hellhammer, D. (Trier)

Review Articles

86 Neuropsychotherapy for Chronic Pain: Changing Pain Memories by Behavioral Interventions Flor, H.; Hermann, C. (Mannheim)

96 Imaginary Reliving in Psychotherapy Modifies Mind and Brain

Schauer, M.; Elbert, T.; Gotthardt, S.; Rockstroh, B.; Odenwald, M.; Neuner, F. (Konstanz)

104 Neural Correlates of Exposure Therapy in Patients Suffering from Specific Phobia

Schienle, A. (Trier/Gießen); Schäfer, A. (Gießen)

112 Neuropsychological Assessment and Therapy of Deficits in Attention and Mnestic Functions in Childhood

Petermann, F.; Lepach, A. (Bremen)

122 Behavioral Medicine, Psychotherapy and Dentistry Meinlschmidt, G.; Bolt, O. (Trier/Basel)

Forum

133 Neuropsychotherapy: Comments of a

Neuropsychologist

Gauggel, S. (Aachen)

Interview

139 Niels Birbaumer: 'The Increasing Biopsychological Knowledge Will Revolutionize the Issue of Indication and Prognosis of Psychotherapy' Hautzinger, M. (Tübingen) 


\section{Verhaltenstherapie}

\section{Band 16, Heft 2, Juni 2006}

141 Neuigkeiten vom Buch- und Zeitschriftenmarkt

146 Fort- und Weiterbildung

151 Mitteilungen der Verbände

156 Tagungen und Kongresse

84 Impressum

159 Hinweise für Autoren

U2 Erläuterungen zum Titelbild (2. Umschlagseite) Leibl, C.; Vogt, B. (Prien/Chiemsee)

Einen Ausblick auf den Inhalt der kommenden Hefte finden Sie auf Seite 160.

\section{Vol. 16, Issue 2, June 2006}

141 Articles and Books

146 Education

151 Information by Behavior Therapy Associations

156 Meetings and Conferences

84 Imprint

159 Guidelines for Authors

C2 Explanations on the Title (Inside front cover) Leibl, C.; Vogt, B. (Prien/Chiemsee)

Forthcoming papers are listed on page 160.

\section{KARGER}

Fax +49 7614520714

E-mail Information@Karger.de

www.karger.com (c) 2006 S. Karger GmbH, Freiburg

Artikel (Volltext) und Inhaltsverzeichnisse

sowie das vorläufige Inhaltsverzeichnis des nächsten Heftes: www.karger.com/ver_bk.htm 